\title{
Outcomes of anal cancer treated with definitive IMRT-based chemoradiation
}

\author{
Michael D. Chuong • Sarah E. Hoffe • Jill Weber • \\ Jessica M. Freilich • Harry Lomas • \\ Khaldoun Almhanna $\cdot$ Sophie Dessureault • \\ William R. Dinwoodie $\cdot$ Richard Kim • \\ Kenneth L. Meredith • Jonathan R. Strosberg • \\ David Shibata • Nikhil G. Rao • Matthew C. Biagioli • \\ Ravi Shridhar
}

Received: 9 April 2012 /Accepted: 23 April 2012 /Published online: 14 May 2012

(C) Springer-Verlag 2012

\begin{abstract}
Objective Despite excellent long-term outcomes, definitive chemoradiation (CRT) for squamous cell carcinoma (SqCC) of the anal canal with traditional radiotherapy techniques results in significant morbidity. Accruing data supports intensity-modulated radiotherapy (IMRT)-based treatment, and we report our institutional experience using this approach.

Methods We reviewed patients with nonmetastatic anal canal SqCC treated with definitive IMRT-CRT. Clinically node-negative patients initially received 36 Gy to the elective pelvic and inguinal lymph nodes and 40 Gy to gross tumor volume (GTV) while node-positive patients received 45 and $50 \mathrm{~Gy}$, respectively. All patients were considered for a GTV boost depending on the degree of clinical response and acute treatment-related toxicity.
\end{abstract}

M. D. Chuong $(\bowtie) \cdot$ S. E. Hoffe · J. M. Freilich • N. G. Rao •

M. C. Biagioli $\cdot$ R. Shridhar

Department of Radiation Oncology, H. Lee Moffitt Cancer Center

\& Research Institute,

12902 Magnolia Drive,

Tampa, FL 33612, USA

e-mail: michael.chuong@moffitt.org

J. Weber $\cdot$ K. Almhanna - S. Dessureault $\cdot$ W. R. Dinwoodie $\cdot$

R. Kim $\cdot$ K. L. Meredith $\cdot$ J. R. Strosberg $\cdot$ D. Shibata

Gastrointestinal Tumor Program,

H. Lee Moffitt Cancer Center \& Research Institute,

Tampa, FL, USA

H. Lomas

University of South Florida College of Medicine,

Tampa, FL, USA
Results We identified 52 patients with T1-4N0-3M0 SqCC of the anal canal. Median follow-up was 21 months (range, 7.7-68.1 months). Two-year locoregional control, overall survival, disease-free survival, distant metastasis-free survival, and colostomy-free survival were 94.6, 100, 82.6, 90, and $94.7 \%$, respectively. Acute grade $3+$ nonhematologic and hematologic toxicities were observed in 21.1 and $63.5 \%$, respectively. No acute grade 4 nonhematologic toxicity was observed.

Conclusion Our series demonstrates that definitive IMRTbased chemoradiation with standard fractionation for anal $\mathrm{SqCC}$ results in excellent outcomes with minimal toxicity.

Keywords Anal cancer · Anal canal · Squamous cell carcinoma $\cdot$ Intensity-modulated radiation therapy $\cdot$ IMRT . Chemoradiation

\section{Objective}

The incidence of squamous cell carcinoma $(\mathrm{SqCC})$ of the anal canal has increased significantly in the USA over the past several decades [1]. Infection by human papilloma virus (HPV), men who have sex with men (MSM), and human immunodeficiency virus (HIV) are established as clear risk factors $[2,3]$. To that extent, SqCC of the anal canal remains a disease that is largely able to be cured by definitive chemoradiation, as initially demonstrated by the seminal work of Nigro et al., with abdominoperineal resection (APR) being reserved for locoregional treatment failure [4-8]. 
Although the historical rates of locoregional control (LRC), overall survival (OS), and colostomy-free survival (CFS) with two- and three-dimensional radiation therapy (RT) approaches are exceptional, these techniques are associated with significant morbidity. The rates of acute grade $3+$ skin and gastrointestinal (GI) toxicities reported from Radiation Therapy Oncology Group (RTOG) 98-11 were 48 and $34 \%$, respectively [9]. Furthermore, RTOG 87-04 reported a $26 \%$ overall rate of grade $4-5$ acute toxicity [10]. Such severe toxicity increases the risk of treatment breaks that may affect patient outcomes. RTOG 92-08 required patients to undergo a 2-week treatment break at $36 \mathrm{~Gy}$, and when compared to historical control data, this resulted in markedly lower 3-year CFS ( 9 vs. $22 \%$ ) [11]. The ability to deliver high-dose radiation to the intended target volume while limiting dose to normal tissues is critical, and the therapeutic ratio can be maximized by using intensity-modulated radiation therapy (IMRT).

The use of IMRT has been well studied in other disease sites [12-14]. Compared to older techniques, IMRT allows for superior dose conformality around target volumes while simultaneously limiting radiation dose to nearby normal structures [15]. Data evaluating IMRT for anal cancer management is limited, but several institutions have published excellent results including OS, LRC, and CFS rates of 88 100, 84-95, and 84-91\%, respectively [16-19]. Moreover, this has been accomplished with low rates of acute grade $3+$ toxicity. Because of the limited published IMRT-based data, we reviewed our institutional experience using definitive IMRT-based chemoradiation in the management of SqCC of the anal canal.

\section{Methods}

\section{Patients}

An institutional review board-approved radiation oncology database was queried for patients with nonmetastatic SqCC of the anal canal treated with definitive IMRT-CRT. Initial staging included a computed tomography (CT) scan of the abdomen and pelvis and 18-fluorodeoxyglucose positron emission tomography (PET)/CT scan. HPV status was not included in the standard workup protocol. Staging was performed according to the American Joint Committee on Cancer (AJCC) Staging Manual, 6th edition [20].

Radiation treatment planning and delivery

CT-based planning was performed for all patients using IV and oral contrast with the patient lying prone on a carbon fiber belly board. One patient with cerebral palsy was simulated and treated in the supine position due to technical difficulties lying prone. Three-millimeter CT slices were obtained from approximately L3 to the mid-femur. Patients with exophytic tumors were simulated with Aquaplast bolus. One patient with a large exophytic tumor was treated with custom-made bolus (dotDecimal, Sanford, FL). Males were instructed to place their genitalia in the inferior position to displace it as much as possible from the field or treatment. A CT-compatible wire was placed on the anal verge and in the gluteal folds for identification on CT scan. All treatment planning was performed using the Pinnacle Treatment Planning System (Philips, Andover, MA). A Pinnacle workstation was used to contour the gross target volume (GTV) and clinical target volume (CTV), with the CTV including elective pelvic and inguinal nodes at risk for microscopic disease involvement. A planning target volume (PTV) was created to account for variation in daily patient setup, consisting of a $0.5-1.0-\mathrm{cm}$ CTV expansion. Target volumes were drawn based on guidelines from the RTOG anorectal contouring atlas [21]. Normal tissue dosing constraints were established for small bowel (maximum 45 $\mathrm{Gy}$, mean $<30 \mathrm{~Gy}$ ), femoral heads (maximum $45 \mathrm{~Gy}$, mean $<30 \mathrm{~Gy}$ ), genitalia (maximum $50 \mathrm{~Gy}$, mean $<30 \mathrm{~Gy}$ ), and bladder (mean $<45 \mathrm{~Gy}$ ).

IMRT was delivered using seven co-planar fields with compensators and 6-15-MeV photons. The PTV was to be covered between 95 and $110 \%$ of the prescription dose. IMRT was delivered in consecutive 1.8- or 2.0-Gy fractions Monday through Friday. Our treatment approach evolved from one initially based on a sequential boost $(n=10)$ to an approach based on a simultaneous integrated boost, also known as dose painting $(n=42)$. For patients treated with a sequential boost to the GTV and pelvic/inguinal nodes, the initial pelvic field extended superiorly to L5/S1 and received 30.6 Gy in 1.8-Gy fractions. This was followed by a 14.4-Gy boost in 1.8-Gy fractions after lowering the top field border to the bottom of the sacroiliac joints. A conformal boost to the GTV alone was then delivered for patients with T3, T4, node-positive, or residual T2 tumors. More recently, patients have been treated with IMRT dose-painting technique. For node-negative patients, $40 \mathrm{~Gy}$ in 2-Gy fractions was delivered to the GTV while the elective nodes simultaneously received 36 Gy in 1.8-Gy fractions. For node-positive patients, 50 Gy in 2-Gy fractions was delivered to the GTV while 45 Gy in 1.8-Gy fractions was delivered to the elective nodes. All patients were considered for a GTV boost to a maximum of approximately $60 \mathrm{~Gy}$ regardless of nodal status. The boost dose was determined based upon the degree of clinical tumor response and treatment-related toxicity.

\section{Chemotherapy}

Two cycles of bolus 5-fluorouracil (5-FU) $1,000 \mathrm{mg} / \mathrm{m}^{2}$ (days 1-4, 29-32) and mitomycin C (MMC) $10 \mathrm{mg} / \mathrm{m}^{2} \mathrm{IV}$ 
bolus (days 1,29 ) were prescribed to be delivered concurrently with RT. One patient was treated with cisplatin $75 \mathrm{mg} / \mathrm{m}^{2}$ (days 1 and 29) and bolus 5-FU because she was being treated concurrently for breast cancer. The number of chemotherapy cycles and dosing were adjusted based upon acute toxicity as determined by the treating medical oncologist.

\section{Follow-up}

All patients were seen at least weekly for evaluation and management of treatment-related toxicities. Followup after treatment completion was performed according to the National Comprehensive Cancer Network guidelines [22]. Acute toxicities were considered to have occurred $\leq 3$ months after chemoradiation completion and late toxicities occurred $>3$ months later. Acute and late toxicities were graded according to the NCI Common Terminology Criteria for Adverse Events, version 4.0 [23].

Kaplan-Meier analysis was performed to evaluate the end points of overall survival (OS), colostomy-free survival (CFS), locoregional control (LRC), disease-free survival (DFS), and distant metastasis-free survival (DMFS). Each end point was calculated from the date of initial biopsy. CFS was defined as the interval to colostomy, LRC was defined from interval to local or regional failure diagnosis, and DFS was defined to the time of locoregional/distant failure or death. DMFS was the interval to distant metastasis diagnosis.

\section{Results}

We identified 52 patients with T1-4N0-3M0 biopsy-proven SqCC of the anal canal who underwent definitive concurrent IMRT-CRT between December 2006 and September 2011. Patient and tumor characteristics are shown in Table 1. The median age at initial presentation was 59 years (range, 3683 years), and the majority had no clinical evidence of nodal involvement (57.7\%). AJCC stage I, II, IIIA, and IIIB disease was present in 19.2, 34.6, 7.7, and $38.5 \%$, respectively. Four patients $(7.7 \%)$ were known to be HIV positive prior to treatment with CD4+ counts $>200$ and were actively taking antiretroviral medication at the time of presentation and during chemoradiation. One patient was chronically immunosuppressed after undergoing a kidney transplant for end-stage renal disease. No patients had previously received chemotherapy or radiation therapy.

Median follow-up was 21 months (range, 7.768.1 months). Two-year LRC, OS, DFS, DMFS, and CFS were 94.6, 100, 82.6, 90, and 94.7\%, respectively (Figs. 1, 2,3 , and 4). No patient was deceased from any cause at the
Table 1 Patient and tumor characteristics

\begin{tabular}{|c|c|}
\hline Demographic & $N(\%)$ \\
\hline \multicolumn{2}{|l|}{ Age } \\
\hline Median (years) & 59 \\
\hline Range & $36-83$ \\
\hline \multicolumn{2}{|l|}{ Gender } \\
\hline Male & $12(23.1)$ \\
\hline Female & $40(76.9)$ \\
\hline \multicolumn{2}{|l|}{ Immunosuppression } \\
\hline HIV+ & $4(7.7)$ \\
\hline Organ transplant & $1(1.9)$ \\
\hline \multicolumn{2}{|l|}{ T stage } \\
\hline $\mathrm{T} 1$ & $12(23.1)$ \\
\hline $\mathrm{T} 2$ & $17(32.7)$ \\
\hline $\mathrm{T} 3$ & $16(30.7)$ \\
\hline $\mathrm{T} 4$ & $7(13.5)$ \\
\hline \multicolumn{2}{|l|}{$\mathrm{N}$ stage } \\
\hline No & $30(57.7)$ \\
\hline N1 & $7(13.5)$ \\
\hline N2 & $10(19.2)$ \\
\hline N3 & $5(9.6)$ \\
\hline \multicolumn{2}{|l|}{ AJCC stage } \\
\hline I & $10(19.2)$ \\
\hline II & $18(34.6)$ \\
\hline IIIA & $4(7.7)$ \\
\hline IIIB & $20(38.5)$ \\
\hline \multicolumn{2}{|l|}{ Grade } \\
\hline 1 & $7(13.5)$ \\
\hline 2 & $16(30.7)$ \\
\hline 3 & $20(38.5)$ \\
\hline X & $9(17.3)$ \\
\hline
\end{tabular}

time of this analysis. Four were diagnosed with distant metastasis $(7.7 \%)$ to the lung $(n=1)$, retroperitoneal lymph nodes $(n=1)$, or liver $(n=2)$. Two developed a locoregional recurrence (3.8\%); one underwent an APR while the other had salvage high-dose rate (HDR) intracavitary brachytherapy, and both currently are without evidence of disease. A second patient with pre-existing chronic diarrhea of unclear etiology had worsening diarrhea during treatment and underwent a colostomy for symptomatic management; this was reversed 2 months later, and the patient is currently disease free.

Radiation treatment parameters are presented in Table 2. Median GTV dose was 56 Gy (range, 40-62.5 Gy). Fortyseven $(90.4 \%)$ patients received a GTV boost (median 11.3 Gy; range, 2-22 Gy) in 2-Gy fractions. The GTV boost dose was determined based upon clinical treatment response and severity of treatment-related toxicity. Because of poor clinical response, two patients received a more aggressive boost at 2.25 Gy per fraction and 2.5 Gy per fraction to a 


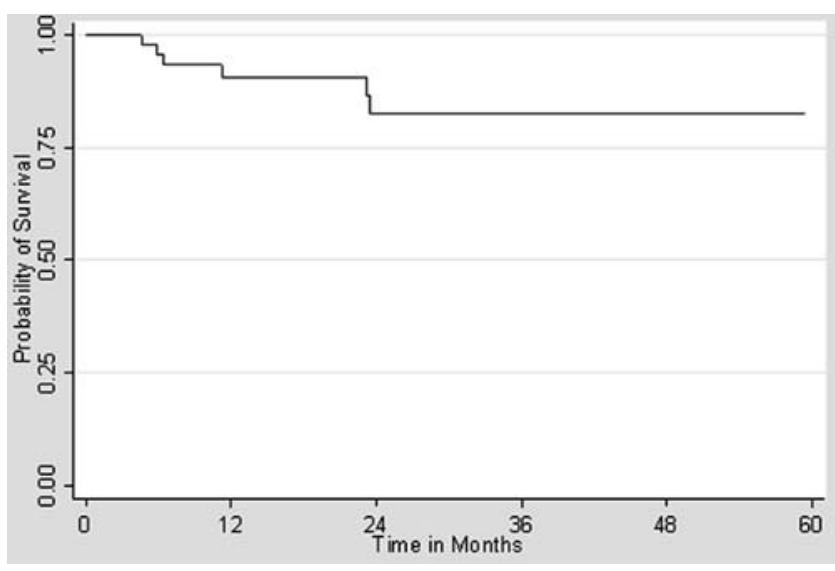

Fig. 1 Three-year disease-free survival

total dose of 61.25 and $62.5 \mathrm{~Gy}$, respectively. Two received an HDR intracavitary brachytherapy boost because of initial vaginal wall invasion. Both patients had a clinical complete response and are currently without evidence of disease. Typical isodose lines for a T2N0M0 patient are illustrated in Fig. 5. The patient later received a GTV boost of 6 Gy for a cumulative GTV dose of 56 Gy.

Treatment was well tolerated. The prescribed radiation dose was delivered in 45 (86.5\%) patients. RT was stopped early in seven (13.5\%) patients at a median dose of 52 Gy (range, 40-60 Gy). The median difference between the delivered and prescribed GTV dose in these patients was 4 Gy (range, 2-6 Gy). The most common reason for early termination of RT was moist perianal desquamation, as is commonly the case in patients treated with conventional RT techniques. Four patients had a treatment break, of which all except one ultimately completed the prescribed course of RT. The median duration of the break was 11 days (range, 6-12 days) and was indicated because of either diarrhea ( $n=$ $1)$, moist desquamation $(n=2)$, or neutropenia $(n=1)$. All patients who had a treatment break ultimately had a

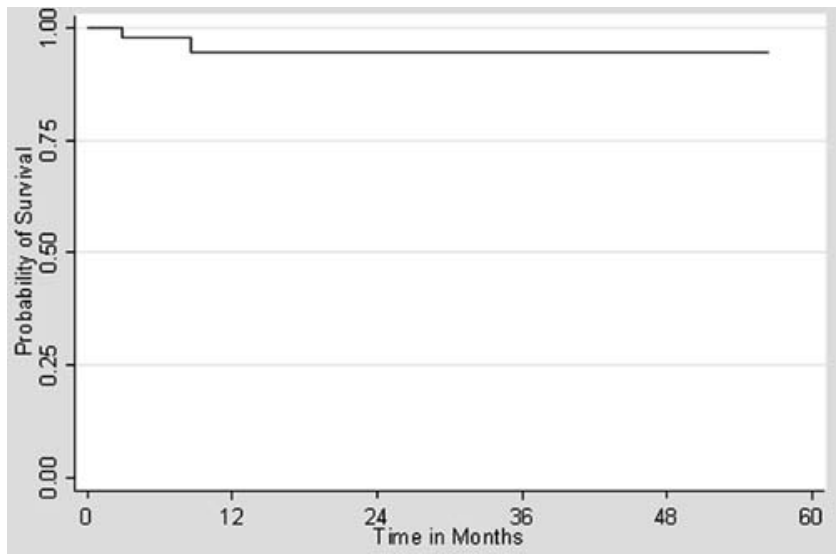

Fig. 2 Three-year locoregional control

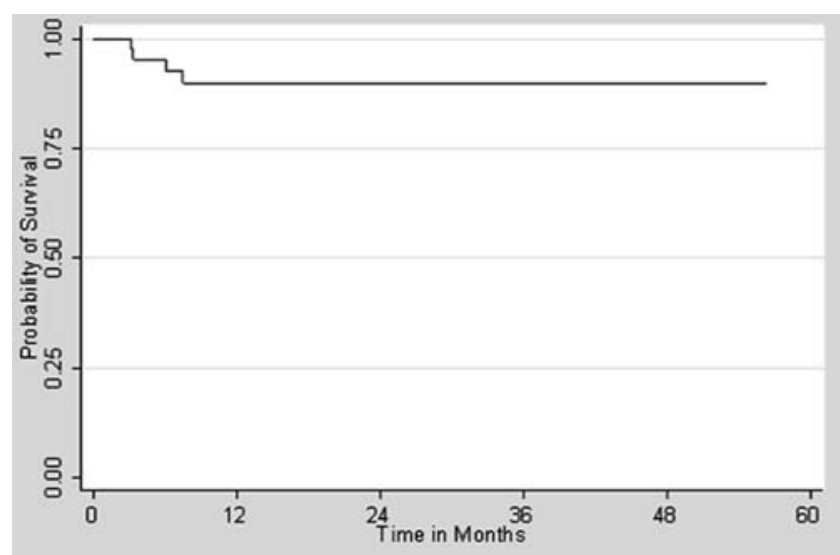

Fig. 3 Three-year distant metastasis-free survival

complete clinical response and are currently without evidence of disease.

The full course of 5-FU and MMC was completed by 22 patients $(42.3 \%)$. Because of treatment-related toxicity, the second cycle of MMC was held in $21(40.4 \%)$ while the second cycle of 5-FU was held in only $4(7.7 \%)$. Dose reduction of 5-FU or MMC during any cycle occurred in 13 $(25 \%)$ and $6(11.5 \%)$, respectively. All patients except for two received at least 1 cycle of 5-FU and MMC; one patient was diagnosed with breast cancer concurrently with anal cancer and cisplatin was substituted for MMC. MMC was withheld in a second patient secondary to chronic renal insufficiency, and 5-FU was given alone. One patient was diagnosed with anal cancer while pregnant and received 1 cycle of 5-FU and MMC while recovering from a Cesarean section delivery, and then completed two additional cycles concurrently with RT for a total of 3 cycles.

Table 3 illustrates the worst acute hematologic and nonhematologic treatment-related toxicities. The overall incidence of acute nonhematologic grade $3+$ toxicity was $21.1 \%$. Acute grade 3 GI, genitourinary (GU), and

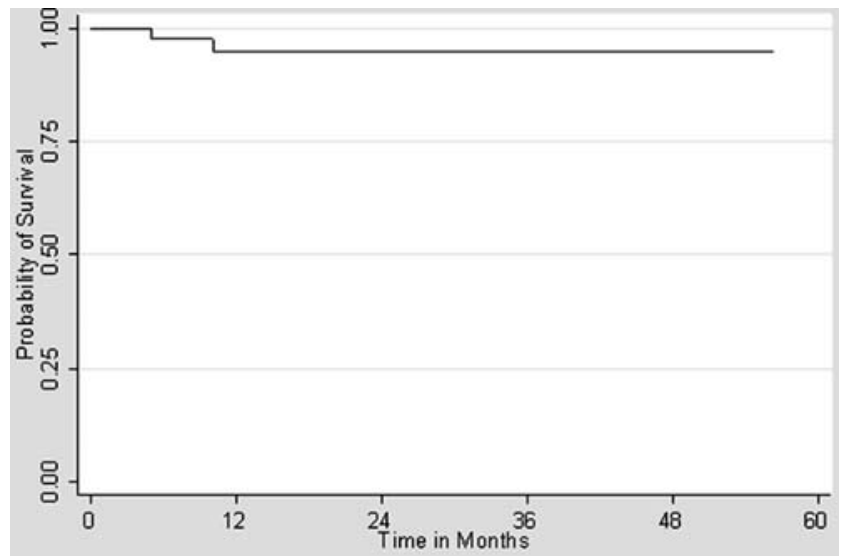

Fig. 4 Three-year colostomy-free survival 
Table 2 Radiotherapy doses delivered to the initial CTV, initial GTV, and boost GTV

\begin{tabular}{lll}
\hline & \multicolumn{2}{c}{ Median dose, Gy (range) } \\
\hline Target volume & $\mathrm{N} 0$ & $\mathrm{~N}+$ \\
GTV & $40(34-50)$ & $50(34-50)$ \\
Initial & 11.2 & 10 \\
Boost & 54.7 & 56 \\
Total & $\mathrm{NA}$ & $10(9-12)^{\mathrm{a}}$ \\
HDR brachytherapy & & \\
CTV & $38(36-45)$ & $45(34-46)$ \\
\hline
\end{tabular}

$N A$ not applicable

a $4 \mathrm{~Gy} \times 3 ; 3 \mathrm{~Gy} \times 3$

dermatologic toxicities were documented in 5 (9.6\%), 0 , and $6(11.5 \%)$ patients, respectively. One patient with grade 3 diarrhea had pre-existing chronic diarrhea of unclear etiology for several years prior to RT, which was exacerbated during treatment and ultimately required a palliative diverting loop colostomy that was eventually reversed. No grade 4 or higher acute nonhematologic toxicity was observed. The incidence of acute hematologic grade 3 and 4 toxicity was 44.3 and $19.2 \%$, respectively.

\section{Discussion}

Chemotherapy delivered concurrently with RT for the definitive treatment of anal $\mathrm{SqCC}$ has significantly reduced the role of surgical resection and need for colostomy, but at the expense of potentially significant RT-related toxicity when traditional RT techniques are used $[6,9,10]$. For instance, $74 \%$ of patients treated in RTOG 98-11 experienced grade 3-4 nonhematologic toxicity while RTOG 87-04 reported a $26 \%$ overall rate of grade $4-5$ acute toxicity $[9,10]$. While IMRT has been more widely adopted in the treatment of other disease sites to reduce dose to normal critical structures, published data have only recently begun accruing in support of its use as standard of care for anal cancer [24-26].

Our data support the use of definitive IMRT-based chemoradiation for anal canal SqCC. This approach results in superior long-term outcomes with markedly less toxicity

Fig. 5 Typical isodose curves for a T2N0M0 anal cancer patient treated with IMRT and dose-painting technique. The GTV received a sequential 6-Gy boost for a cumulative dose of 56 Gy
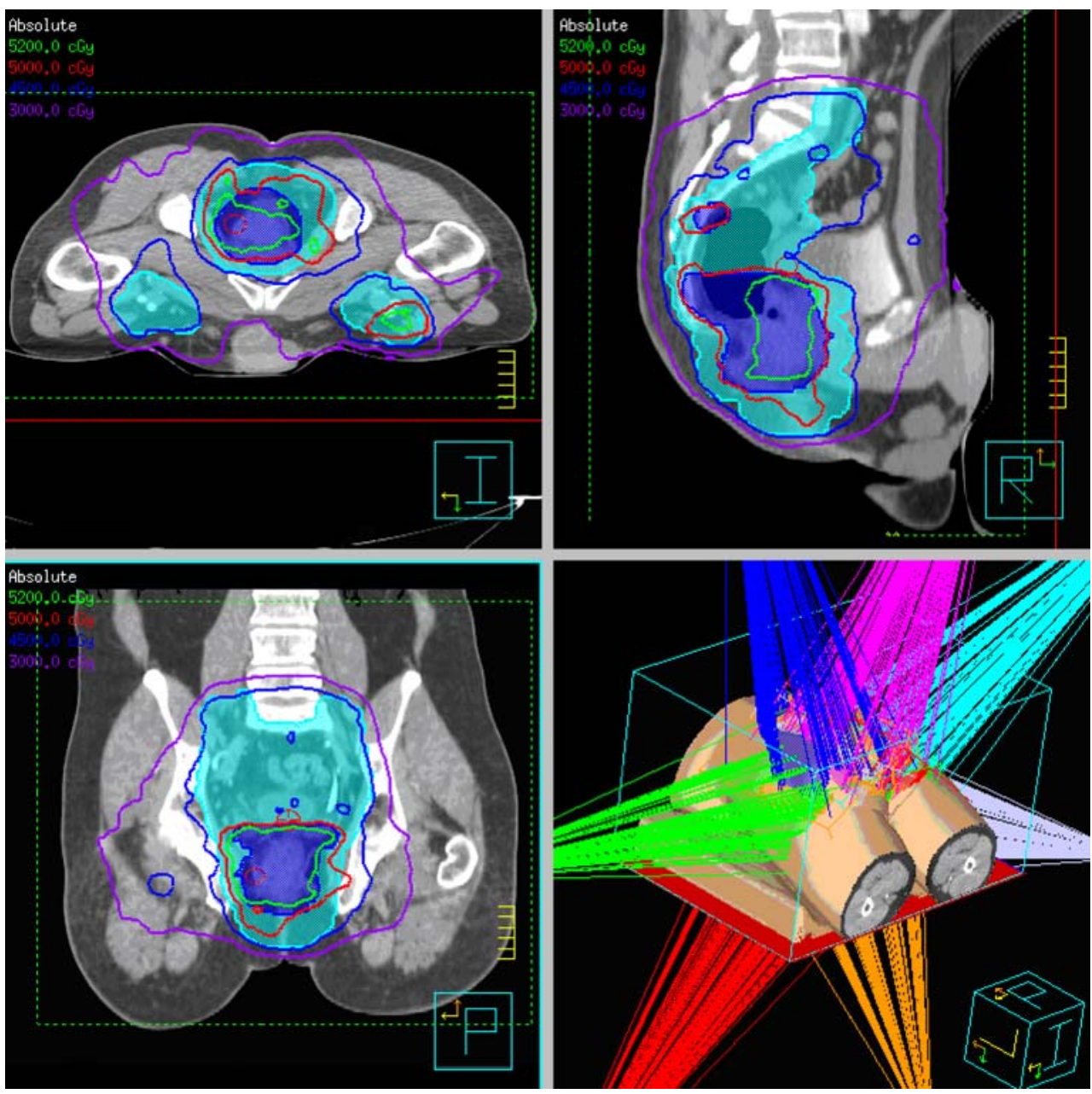
Table 3 Worst acute nonhematologic and hematologic toxicity

\begin{tabular}{lrcll}
\hline Toxicity & \multicolumn{3}{l}{ Grade (\%) } & \\
\cline { 2 - 5 } & \multicolumn{1}{l}{1} & 2 & 3 & 4 \\
\hline Gastrointestinal (GI) & $11(21.2)$ & $18(34.6)$ & $5(9.6)$ & 0 \\
Genitourinary (GU) & $10(19.2)$ & $4(7.7)$ & \multicolumn{1}{l}{0} & 0 \\
Skin & $11(21.2)$ & $19(36.5)$ & $6(11.5)$ & 0 \\
Anemia & $6(11.5)$ & $5(9.6)$ & $7(13.5)$ & 0 \\
Leukopenia & $10(19.2)$ & $5(9.6)$ & $9(17.3)$ & $6(11.5)$ \\
Thrombocytopenia & $7(13.5)$ & $4(7.7)$ & $7(13.5)$ & $4(7.7)$ \\
\hline
\end{tabular}

than either conventional or 3D conformal techniques. Twoyear LRC, OS, DFS, DMFS, and CFS were 94.6, 100, 82.6, 90 , and $94.7 \%$, respectively. In addition to excellent clinical outcomes, we observed rates of significant acute toxicity similar to that of other published series. Our acute grade $3+$ nonhematologic and hematologic toxicity rates were 21.1 and $59.9 \%$, respectively. Nearly all patients were able to complete treatment without interruption; only four $(7.7 \%)$ required a treatment break of a median 11 days (range, 612 days). The low incidence of significant acute toxicity reported in this study is notable especially when considering that most patients were treated with dose-painting technique in up to 2 Gy fractions. Previously published IMRT experiences did not use fraction sizes larger than 1.8 Gy with the exception of Salama et al. [18].

Our data are similar to previously published IMRT-based anal cancer experiences, as demonstrated in Table 4. These data compare very favorably to clinical outcomes and toxicity as reported from the pre-IMRT era. Salama et al. reported the outcomes of 53 patients treated with concurrent IMRT and chemotherapy [18]. With median follow-up of 14.5 months, the 18-month LRC, freedom from distant failure, CFS, and OS were 83.9, 92.9, 83.75, and $93.4 \%$, respectively. Acute grade 3+ toxicity was reported in $38 \%$ with none experiencing grade 4 complications. However, over $40 \%$ of patients required a treatment break, albeit for a short duration (median 4 days). Kachnic et al. published a multi-institutional experience from Boston Medical Center (BMC) and Massachusetts General Hospital (MGH) of 43 patients with 2-year LRC, OS, CFS, and DMFS of 95, 94, 90 , and $92 \%$, respectively [16]. The incidence of acute grade 3+ GI, GU, and dermatologic toxicities were 7, 7, and $10 \%$. The dose prescription depended on the tumor stage, with the GTV receiving from 50 to 54 Gy in 1.8-Gy fractions. Bazan et al. were the first to directly compare conventional RT (37\%) and IMRT (63\%) in a series of 45 patients, with the results clearly favoring IMRT [27]. IMRT resulted in 3-year OS, LRC, and progression-free survival of $87.8,91.9$, and $84.2 \%$, respectively, compared

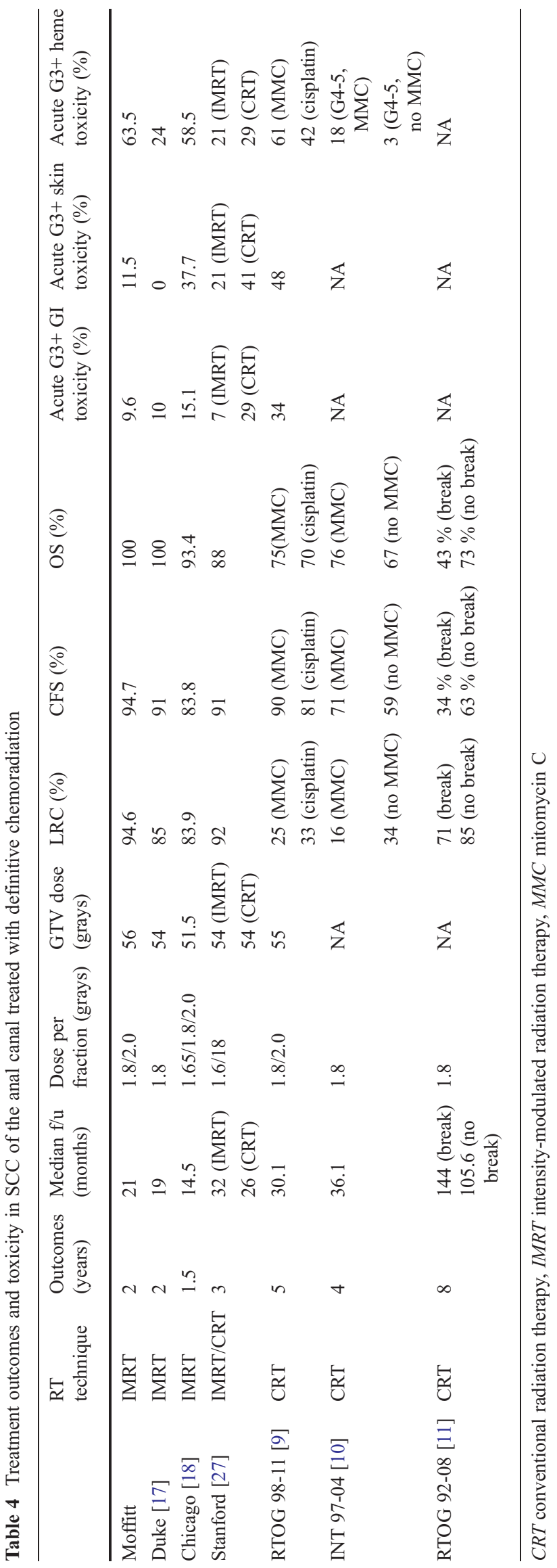


to $51.8,56.7$, and $56.7 \%$, respectively, for the conventional group (all $p<0.01$ ). Patients treated with a conventional approach were treated over a longer duration (57 vs. 40 days, $p<0.0001$ ), required more treatment breaks ( 88 vs. $34.5 \%$, $p=0.001$ ), and longer treatment breaks (12 vs. 1.5 days, $p<0.0001)$. Dosimetric parameters were improved with IMRT, including mean bowel volume $>35$ Gy being reduced by $27 \%$. Although the vast majority of published IMRT-based data for anal cancer are retrospective, early prospective outcomes from RTOG 05-29, a phase II multi-institutional trial, were recently reported [28]. Two-year locoregional failure, OS, DFS, and CFS were $20,88,77$, and $86 \%$, respectively. These are similar to previously published data as well as our own institutional experience. Prolonged patient follow-up in this study will likely continue to demonstrate excellent results.

Our study population included a higher percentage of patients with locally advanced disease, particularly those with stage III tumors, when compared to other anal IMRT series. On the same note, the incidence of stage II tumors in our series was somewhat lower than in other series. The incidence of stage I/II/III disease in the Moffitt, Stanford, Duke, Chicago, and BMC/MGH series was 19.2/34.6/46.2, 7/59/34, 13/36/31, $7.6 / 58.5 / 34$, and $16 \% / 37 \% / 42 \%$, respectively.

The published literature suggests that treatment breaks are associated with inferior local control and survival for anal cancer patients $[10,29]$. Four patients in our study had a break, receiving a median total GTV dose of 59 Gy (range, 50-61 Gy). All eventually had a complete clinical response and currently have no evidence of disease. Some studies have not shown an association between treatment breaks with worse clinical outcomes, including a pooled data analysis of RTOG 87-04 and 98-11 [30, 31]. Although the effect of a treatment break is unclear, an uninterrupted treatment course should be delivered if possible.

\section{Conclusion}

Our series demonstrates that definitive IMRT-based chemoradiation with standard fractionation for anal $\mathrm{SqCC}$ results in excellent outcomes and minimal toxicity. This study supports the emerging role of IMRT as the new standard of care.

\section{References}

1. Johnson L, Madeleine M, Newcomer L, Schwartz S, Daling J (2004) Anal cancer incidence and survival: the surveillance, epidemiology, and end results experience, 1973-2000. Cancer 101 (2):281-288

2. Palefsky J (1994) Anal human papillomavirus infection and anal cancer in HIV-positive individuals: an emerging problem. AIDS 8 (3):283
3. Nyitray AG, Carvalho da Silva RJ, Baggio ML, Smith D, Abrahamsen M, Papenfuss M, Lin HY, Quiterio M, Salmeron J, Lazcano-Ponce E, Villa LL, Giuliano AR (2011) Six-month incidence, persistence, and factors associated with persistence of anal human papillomavirus in men: the HPV in men study. J Infect Dis. doi:10.1093/infdis/jir637

4. Anonymous (1996) Epidermoid anal cancer: results from the UKCCCR randomised trial of radiotherapy alone versus radiotherapy, 5-fluorouracil, and mitomycin. UKCCCR Anal Cancer Trial Working Party. UK Co-ordinating Committee on Cancer Research. Lancet 348(9034):1049-1054

5. Nigro N, Seydel H, Considine B, Vaitkevicius V, Leichman L, Kinzie J (1983) Combined preoperative radiation and chemotherapy for squamous cell carcinoma of the anal canal. Cancer 51(10):18261829

6. Nigro N, Vaitkevicius V, Considine B (1993) Combined therapy for cancer of the anal canal: a preliminary report. Dis Colon Rectum 36 (7):709-711

7. Bartelink H, Roelofsen F, Eschwege F, Rougier P, Bosset J, Gonzalez D, Peiffert D, Van Glabbeke M, Pierart M (1997) Concomitant radiotherapy and chemotherapy is superior to radiotherapy alone in the treatment of locally advanced anal cancer: results of a phase III randomized trial of the European Organization for Research and Treatment of Cancer Radiotherapy and Gastrointestinal Cooperative Groups. J Clin Oncol 15(5):2040

8. Nigro N (1984) An evaluation of combined therapy for squamous cell cancer of the anal canal. Dis Colon Rectum 27(12):763-766

9. Ajani JA, Winter KA, Gunderson LL, Pedersen J, Benson AB 3rd, Thomas CR Jr, Mayer RJ, Haddock MG, Rich TA, Willett C (2008) Fluorouracil, mitomycin, and radiotherapy vs fluorouracil, cisplatin, and radiotherapy for carcinoma of the anal canal: a randomized controlled trial. JAMA 299(16):1914-1921. doi:10.1001/ jama.299.16.1914

10. Flam M, John M, Pajak TF, Petrelli N, Myerson R, Doggett S, Quivey J, Rotman M, Kerman H, Coia L, Murray K (1996) Role of mitomycin in combination with fluorouracil and radiotherapy, and of salvage chemoradiation in the definitive nonsurgical treatment of epidermoid carcinoma of the anal canal: results of a phase III randomized intergroup study. J Clin Oncol 14(9):2527-2539

11. John M, Pajak T, Flam M, Hoffman J, Markoe A, Wolkov H, Paris $\mathrm{K}$ (1996) Dose escalation in chemoradiation for anal cancer: preliminary results of RTOG 92-08. Cancer J Sci Am 2(4):205-211

12. Grills IS, Yan D, Martinez AA, Vicini FA, Wong JW, Kestin LL (2003) Potential for reduced toxicity and dose escalation in the treatment of inoperable non-small-cell lung cancer: a comparison of intensity-modulated radiation therapy (IMRT), 3D conformal radiation, and elective nodal irradiation. Int J Radiat Oncol Biol Phys 57(3):875-890

13. Zelefsky MJ, Fuks Z, Happersett L, Lee HJ, Ling CC, Burman CM, Hunt M, Wolfe T, Venkatraman E, Jackson A (2000) Clinical experience with intensity modulated radiation therapy (IMRT) in prostate cancer. Radiother Oncol 55(3):241-249

14. Landry JC, Yang GY, Ting JY, Staley CA, Torres W, Esiashvili N, Davis LW (2002) Treatment of pancreatic cancer tumors with intensity-modulated radiation therapy (IMRT) using the volume at risk approach (VARA): employing dose-volume histogram (DVH) and normal tissue complication probability (NTCP) to evaluate small bowel toxicity. Med Dosim 27(2):121-129

15. Chen YJ, Liu A, Tsai PT, Vora NL, Pezner RD, Schultheiss TE, Wong JY (2005) Organ sparing by conformal avoidance intensitymodulated radiation therapy for anal cancer: dosimetric evaluation of coverage of pelvis and inguinal/femoral nodes. Int J Radiat Oncol Biol Phys 63(1):274-281. doi:10.1016/j.ijrobp.2005.05.052

16. Kachnic LA, Tsai HK, Coen JJ, Blaszkowsky LS, Hartshorn K, Kwak EL, Willins JD, Ryan DP, Hong TS (2010) Dose-painted intensity-modulated radiation therapy for anal cancer: a multi- 
institutional report of acute toxicity and response to therapy. Int $\mathrm{J}$ Radiat Oncol Biol Phys. doi:10.1016/j.ijrobp.2010.09.030

17. Pepek JM, Willett CG, Wu QJ, Yoo S, Clough RW, Czito BG (2010) Intensity-modulated radiation therapy for anal malignancies: a preliminary toxicity and disease outcomes analysis. Int J Radiat Oncol Biol Phys 78(5):1413-1419. doi:10.1016/ j.ijrobp.2009.09.046

18. Salama JK, Mell LK, Schomas DA, Miller RC, Devisetty K, Jani AB, Mundt AJ, Roeske JC, Liauw SL, Chmura SJ (2007) Concurrent chemotherapy and intensity-modulated radiation therapy for anal canal cancer patients: a multicenter experience. J Clin Oncol 25(29):4581-4586. doi:10.1200/JCO.2007.12.0170

19. Milano MT, Jani AB, Farrey KJ, Rash C, Heimann R, Chmura SJ (2005) Intensity-modulated radiation therapy (IMRT) in the treatment of anal cancer: toxicity and clinical outcome. Int J Radiat Oncol Biol Phys 63(2):354-361. doi:10.1016/j.ijrobp.2005.02.030

20. AJCC (2002) AJCC cancer staging manual, 6th edn. Springer, New York

21. Myerson RJ, Garofalo MC, El Naqa I, Abrams RA, Apte A, Bosch WR, Das P, Gunderson LL, Hong TS, Kim JJ, Willett CG, Kachnic LA (2009) Elective clinical target volumes for conformal therapy in anorectal cancer: a radiation therapy oncology group consensus panel contouring atlas. Int J Radiat Oncol Biol Phys 74(3):824830. doi:10.1016/j.ijrobp.2008.08.070

22. National Comprehensive Cancer Network (2011) National Comprehensive Cancer Network clinical practice guidelines. http:// www.nccn.org/professionals/physician_gls/pdf/anal.pdf

23. National Cancer Institute (2010) National Cancer Institute Common Terminology Criteria for Adverse Events (CTCAE) version 4.0. $\mathrm{http}$ ://ctep.cancer.gov/protocolDevelopment/electronic_applications/ ctc.htm

24. Eisbruch A (2009) Radiotherapy: IMRT reduces xerostomia and potentially improves QoL. Nat Rev Clin Oncol 6(10):567-568. doi:10.1038/nrclinonc.2009.143
25. Fenoglietto P, Laliberte B, Allaw A, Ailleres N, Idri K, Hay MH, Moscardo CL, Gourgou S, Dubois JB, Azria D (2008) Persistently better treatment planning results of intensity-modulated (IMRT) over conformal radiotherapy (3D-CRT) in prostate cancer patients with significant variation of clinical target volume and/or organs-at-risk. Radiother Oncol 88(1):77-87. doi:10.1016/j.radonc.2007.12.011

26. Lindsey H (2006) Positive long-term outcomes for IMRT in prostate cancer. Lancet Oncol 7(11):895

27. Bazan JG, Hara W, Hsu A, Kunz PA, Ford J, Fisher GA, Welton ML, Shelton A, Kapp DS, Koong AC, Goodman KA, Chang DT (2011) Intensity-modulated radiation therapy versus conventional radiation therapy for squamous cell carcinoma of the anal canal. Cancer. doi:10.1002/cncr.25901

28. Kachnic LA, Winter KA, Myerson R, Goodyear MD, Willins JD, Esthappan J, Haddock MG, Rotman M, Parikh PJ, Willett C (2011) Two-year outcomes of RTOG 0529: a phase II evaluation of dosepainted IMRT in combination with 5-fluorouracil and mitomycin$\mathrm{C}$ for the reduction of acute morbidity in carcinoma of the anal canal. J Clin Oncol 29:suppl 4, abstr 368

29. Roohipour R, Patil S, Goodman KA, Minsky BD, Wong WD, Guillem JG, Paty PB, Weiser MR, Neuman HB, Shia J, Schrag D, Temple LK (2008) Squamous-cell carcinoma of the anal canal: predictors of treatment outcome. Dis Colon Rectum 51(2):147153. doi:10.1007/s10350-007-9125-Z

30. Ben-Josef E, Moughan J, Ajani JA, Flam M, Gunderson L, Pollock J, Myerson R, Anne R, Rosenthal SA, Willett C (2010) Impact of overall treatment time on survival and local control in patients with anal cancer: a pooled data analysis of Radiation Therapy Oncology Group trials 87-04 and 98-11. J Clin Oncol 28(34):5061-5066. doi:10.1200/JCO.2010.29.1351

31. Meyer A, Meier Zu Eissen J, Karstens JH, Bremer M (2006) Chemoradiotherapy in patients with anal cancer: impact of length of unplanned treatment interruption on outcome. Acta Oncol 45 (6):728-735. doi:10.1080/02841860600726729 